\title{
Simultaneous Use of MutS and RecA for Suppression of Nonspecific Amplification during PCR
}

\author{
Kenji Fukui ${ }^{1,2,3}$ and Seiki Kuramitsu ${ }^{1,3}$ \\ ${ }^{1}$ RIKEN SPring-8 Center, Harima Institute, 1-1-1 Kouto, Sayo-cho, Sayo-gun, Hyogo 679-5148, Japan \\ ${ }^{2}$ Science and Technology Entrepreneurship Laboratory, Osaka University, Suita, Osaka 565-0871, Japan \\ ${ }^{3}$ Department of Biological Sciences, Graduate School of Science, Osaka University, 1-1 Machikaneyama-cho, Toyonaka, \\ Osaka 560-0043, Japan
}

Correspondence should be addressed to Seiki Kuramitsu; kuramitu@bio.sci.osaka-u.ac.jp

Received 27 March 2013; Accepted 21 June 2013

Academic Editor: Janusz M. Bujnicki

Copyright $\odot 2013$ K. Fukui and S. Kuramitsu. This is an open access article distributed under the Creative Commons Attribution License, which permits unrestricted use, distribution, and reproduction in any medium, provided the original work is properly cited.

\begin{abstract}
Thermus thermophilus MutS, a thermostable mismatch-recognizing protein, is utilized in PCR to suppress nonspecific amplification by preventing synthesis from mismatched primers. T. thermophilus RecA also decreases nonspecific amplification by promoting proper hybridization between the primer and template. We observed that MutS and RecA function under the same reaction conditions and that MutS and RecA do not preclude each other. Furthermore, there were some DNA sequences for which only one of the 2 proteins effectively suppressed nonspecific amplification. The simultaneous use of MutS and RecA is a more attractive error-suppressing technique than the use of either of the 2 proteins alone.
\end{abstract}

\section{Introduction}

PCR-based technologies are essential for a wide range of biosciences, ranging from basic life sciences to medical diagnoses [1-3]. The efficiency and reliability of those technologies are often decreased by nonspecific amplifications resulting from mishybridization of primers. Recently, we developed a technique to suppress nonspecific amplification by adding a thermostable mismatch-recognizing protein Thermus thermophilus MutS (ttMutS) in PCR mixture [4]. MutS is a DNA mismatch repair protein, which recognizes a variety of DNA mismatches such as base-base mismatches and base insertion loops $[5,6]$. MutS is thought to bind tightly to the mismatched bases that are generated by mishybridization between primer and template to block the association of a DNA polymerase. Before we established the MutS-based error-suppressing technique, it had been reported that $T$. thermophilus RecA (ttRecA) protein can be used to decrease nonspecific amplification during PCR, which drastically enhances the accuracy of multiplex PCR [7]. RecA is known to be a key enzyme in homologous recombination of DNA molecules, which catalyzes pairing of
2 homologous sequences [8]. The ttRecA protein enhances the proper pairing between the primer and template to suppress nonspecific amplification. Thus, the mechanisms by which ttMutS and ttRecA suppress nonspecific amplification are quite different. In this study, we investigated the difference in the effects and properties of ttMutS and ttRecA in order to evaluate the possibility of simultaneous use of both proteins.

\section{Materials and Methods}

2.1. Overexpression and Purification of Proteins. Thermus thermophilus MutS (ttMutS) was prepared as previously described [4]. A DNA fragment-expressing T. thermophilus RecA (ttRecA) was generated by PCR using the T. thermophilus HB8 genomic DNA as a template. The following primer pairs were used for amplification of the fragment: $5^{\prime}$ ATATCATATGGACGAGAGCAAGCGCAAGGC- $3^{\prime}$ and $5^{\prime}$-ATATGGATCCTTATTACTCCCCCTCGTCCTCGCCC- $3^{\prime}$. The forward and reverse primers contained NdeI and BamHI restriction sites, respectively (underlined). The amplified fragment was ligated into the NdeI and BamHI 
sites of pET-11a (Novagen, Madison, WI, USA) to obtain pET-11a/T. thermophilus recA. Sequence analysis revealed that the construction was error-free. E. coli BL21 (DE3) was transformed with pET-11a/T. thermophilus recA and then grown at $37^{\circ} \mathrm{C}$ in 1.5 liters of YT medium containing $50 \mu \mathrm{g} / \mathrm{mL}$ of ampicillin. When the density of cultures reached $2.9 \times 10^{8}$ cells $/ \mathrm{mL}$, isopropyl $\beta$-D-thiogalactopyranoside was added to $100 \mu \mathrm{M}$. The cells were grown at $37^{\circ} \mathrm{C}$ for $3 \mathrm{~h}$ after induction and harvested by centrifugation. The cells were lysed by sonication in $50 \mathrm{mM}$ Tris- $\mathrm{HCl}(\mathrm{pH} 7.5)$ containing $100 \mathrm{mM} \mathrm{NaCl}$ and $1.5 \mathrm{M} \mathrm{KCl}$ and then heated to $60^{\circ} \mathrm{C}$ for $60 \mathrm{~min}$. After centrifugation at $48,000 \times \mathrm{g}$ for $20 \mathrm{~min}$, the supernatant was isolated, and ammonium sulfate was added to a final concentration of $1.5 \mathrm{M}$. The solution was loaded onto a TOYOPEARL-Phenyl column $(12 \mathrm{~mL}$, TOSOH, Tokyo, Japan) preequilibrated with $50 \mathrm{mM}$ Tris- $\mathrm{HCl}$ ( $\mathrm{pH}$ 8.0) containing $1.5 \mathrm{M}$ ammonium sulfate. The column was washed with the same buffer and then eluted with 1.5$0 \mathrm{M}$ ammonium sulfate gradient in the same buffer. The fraction containing ttRecA was detected by SDS-PAGE and dialyzed against $50 \mathrm{mM}$ Tris- $\mathrm{HCl}(\mathrm{pH} \mathrm{8.0)}$. The solution was loaded onto a TOYOPEARL-DEAE column ( $12 \mathrm{~mL}$, TOSOH) preequilibrated with $50 \mathrm{mM}$ Tris- $\mathrm{HCl}(\mathrm{pH} 8.0)$. The column was washed with the same buffer and then eluted with $0-1.5 \mathrm{M}$ $\mathrm{KCl}$ gradient in $50 \mathrm{mM}$ Tris- $\mathrm{HCl}(\mathrm{pH} 8.0)$.

2.2. PCR Using ttRecA and ttMutS Proteins. The $5^{\prime}$-terminal 423-bp region of the thal806 gene was amplified using the primers $5^{\prime}$-GAGACCACCCGTAGGCGGCT- $3^{\prime}$ and $5^{\prime}$-CTTAAGGGGCCTCGCGCTCT-3'; a 1,278-bp region of the tha1548 gene was amplified using $5^{\prime}$-GAGGAGGTGCTCTACGTGGGCAAGGCC- $3^{\prime}$ and $5^{\prime}$-GGGAAGGTCCTTGAGGCTTCCCGTGTAGC-3'; a 611-bp region of the ttha0122 gene was amplified using $5^{\prime}$-ATGTTCCTGAGGATAGACCGCC- $3^{\prime}$ and $5^{\prime}$-ATCTCCACCCCGGTGAGGC$3^{\prime}$; and a 466-bp region of the tha1300 gene was amplified using $5^{\prime}$-ATGCCCGCCATGGAAGTGG- $3^{\prime}$ and $5^{\prime}$-TGAGCGCCTTCAGGGCCT- $3^{\prime}$. The primers were synthesized by BEX Co. (Tokyo, Japan). The reactions were performed in $1 \times$ Takara GC I buffer (Takara, Shiga, Japan) containing 0.06 units $/ \mu \mathrm{L}$ Takara LA Taq (Takara), $5 \mathrm{ng} / \mu \mathrm{L} T$. thermophilus HB8 genomic DNA, $400 \mathrm{nM}$ primers, $100 \mu \mathrm{M}$ $\mathrm{CoCl}_{2}$, and $400 \mu \mathrm{M}$ dATP, dTTP, dCTP, and dGTP (Takara) in the presence of various concentrations of ttRecA and ttMutS. Thirty PCR cycles were run using ASTEC OC707 (ASTEC, Tokyo, Japan): denaturation step at $95^{\circ} \mathrm{C}$ for $1 \mathrm{~min}$, annealing at $58^{\circ} \mathrm{C}$ for $1 \mathrm{~min}$, and extension at $70^{\circ} \mathrm{C}$ for $2 \mathrm{~min}$ for amplification of the tha1806 gene; and denaturation step at $95^{\circ} \mathrm{C}$ for $1 \mathrm{~min}$, annealing at $50^{\circ} \mathrm{C}$ for $1 \mathrm{~min}$, and extension at $70^{\circ} \mathrm{C}$ for $2 \mathrm{~min}$ for amplification of the tha0122, tha1300, and tha 1548 genes. A $10 \mu \mathrm{L}$ reaction solution was mixed with $1 \mu \mathrm{L}$ of loading buffer $(50 \%[\mathrm{v} / \mathrm{v}]$ glycerol, $0.9 \%[\mathrm{w} / \mathrm{v}]$ SDS, and $0.05 \%[\mathrm{w} / \mathrm{v}]$ bromophenol blue) and electrophoresed on a $1.5 \%$ agarose gel in $1 \times$ TBE buffer. The gel was stained with ethidium bromide, and the products were visualized under ultraviolet light at $254 \mathrm{~nm}$. The relative amounts of the amplified DNA products were quantified using the ImageJ software [10].

\section{Results and Discussion}

As has been previously described, RecA catalyzes the homologous pairing of DNA molecules in an ATP-dependent manner [11-13], and ttRecA requires ATP for the errorsuppressing effect during PCR [7]. On the other hand, ATP is not essential for the error-suppressing effect of ttMutS during PCR [4]. However, it is known that MutS has a Walker's A-type ATPase motif $[14,15]$ and that the conformation and function of MutS are greatly affected by the binding and hydrolysis of ATP. Binding of ATP triggers the formation of sliding-clamp mode of MutS, and enhances the dissociation of MutS from a mismatch $[10,16]$. Therefore, we examined whether or not ATP interferes with the error-suppressing effect of MutS during PCR. The 423base pair (bp) region of the tha1806 gene was amplified by Takara LA Taq using T. thermophilus HB8 genomic DNA as the template. Without ttMutS and ttRecA, 1000to 5000-bp DNA fragments were amplified nonspecifically (Figure 1(c)). ttMutS showed a significant suppressing effect on the nonspecific amplifications in the presence of 0 to $0.4 \mathrm{mM}$ ATP (Figure $1(\mathrm{c})$ ). The previous reports described that $0.4 \mathrm{mM}$ ATP is enough to ensure the maximum effect of ttRecA [7], which was also supported by our results (Figure 1(c)). It also should be noted that $0.4 \mathrm{mM}$ ATP did not influence the specificity of PCR amplification (Figure 1(c), left panel). Thus, ttMutS and ttRecA function under the same reaction conditions. We also demonstrated that $0.4 \mu \mathrm{M}$ ttRecA and $0.8 \mu \mathrm{M}$ ttMutS were suitable for suppressing the nonspecific amplifications during PCR, with a wide range of template concentrations ( 0.9 to $24 \mathrm{ng} / \mu \mathrm{L}$ ) (Figure $1(\mathrm{~d})$ ). Since ttMutS and ttRecA are not stable at the temperature over $90^{\circ} \mathrm{C}$, the error-suppressing effect may be improved by supplementing the reaction with additional proteins at the middle of the PCR cycle. However, as shown in our data, we were able to obtain satisfactory effect without the supplementation when we use $0.4 \mu \mathrm{M}$ ttRecA and $0.8 \mu \mathrm{M}$ ttMutS. The supplementation of the additional proteins may be required when we cannot obtain enough effect under these conditions.

To determine whether ttRecA and ttMutS preclude the effect of each other, a 611-bp region of the tha0122 gene was amplified by Takara LA Taq using T. thermophilus HB8 genomic DNA as a template. In the absence of ttRecA and ttMutS, relatively weak amplification of the target sequence and the nonspecific amplification of 1000- to 1500-bp were observed (Figure 2(a)). Both ttRecA and ttMutS enhanced the amplification of the desired fragment and suppressed the nonspecific amplification (Figure 2(a)). The error-suppressing effect was retained when both ttRecA and ttMutS were present (Figure 2(a)). The same result was also obtained when the 423-bp region of the ttha1806 gene was amplified in the presence of ttRecA and aqMutS (Figure 2(b)). These findings suggest that ttRecA and ttMutS can be used simultaneously without any interfering effects.

In order to evaluate the validity of the simultaneous use of ttRecA and ttMutS, we investigated the difference in the specificities of ttRecA and ttMutS towards the sequences to 


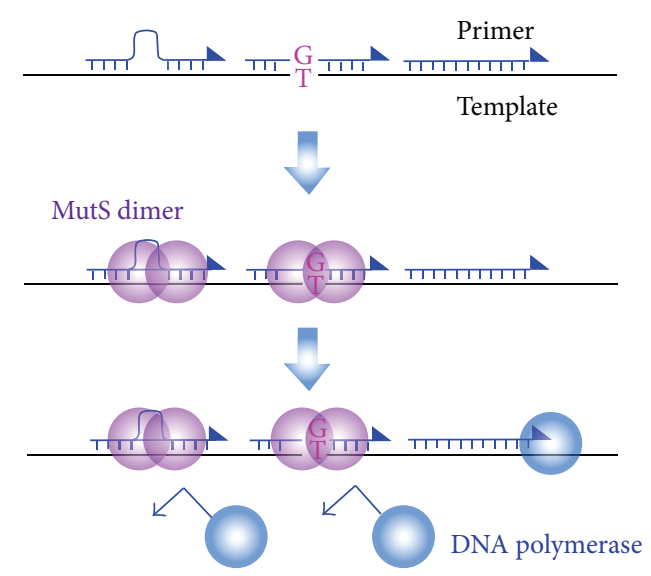

(a)

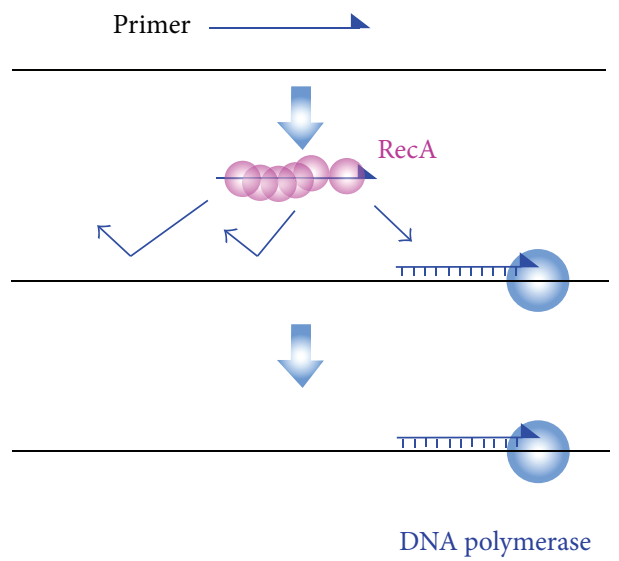

(b)

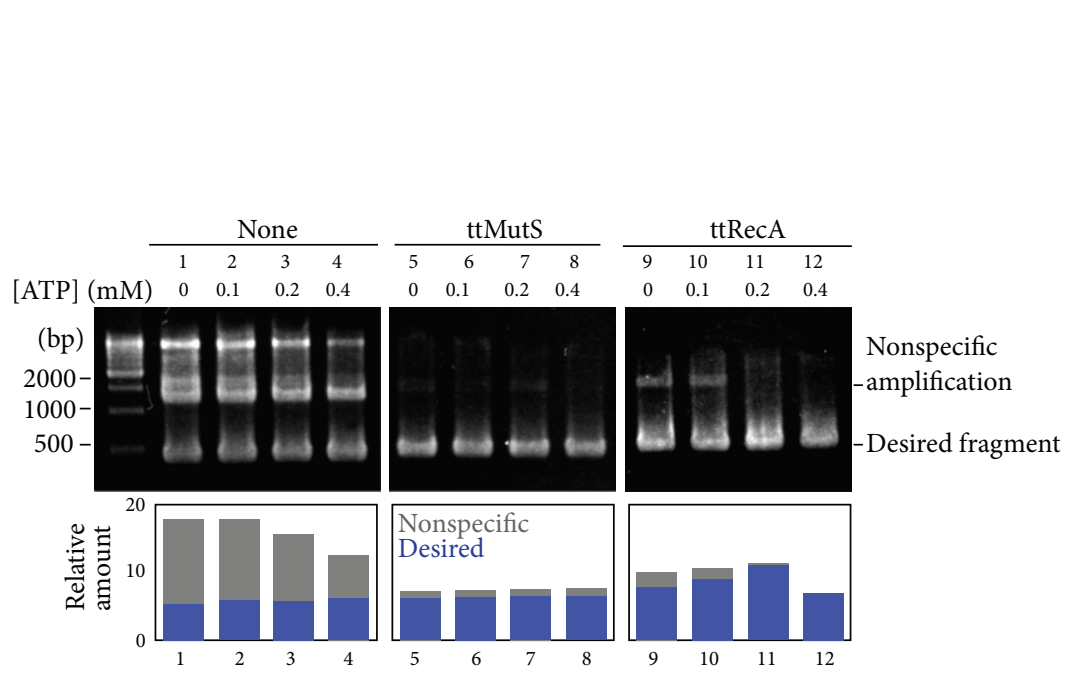

(c)

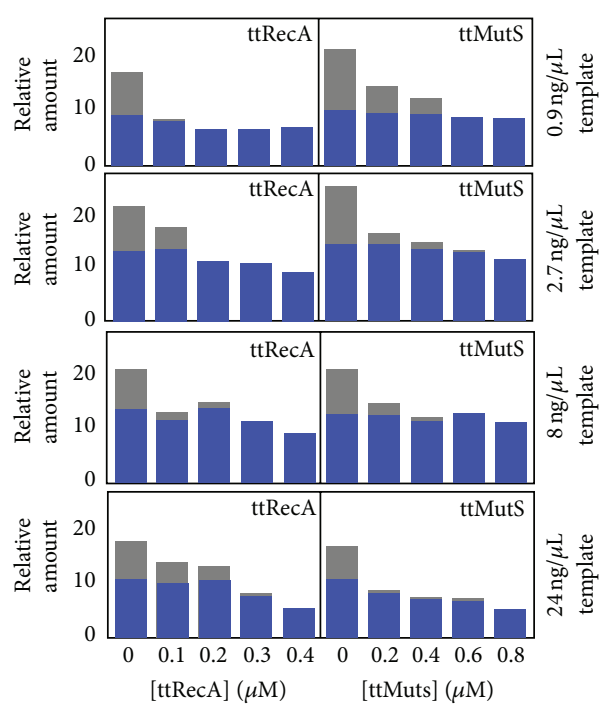

(d)

FIgURE 1: The error-suppressing effects of ttRecA and ttMutS in the presence of ATP. (a) A schematic representation for the mechanism by which ttMutS suppresses nonspecific amplifications during PCR. A ttMutS dimer recognizes mismatched bases generated by mishybridization of the primer and blocks the approach of DNA polymerase. (b) A schematic representation for the mechanism by which ttRecA suppresses nonspecific amplification during PCR. ttRecA promotes proper priming for PCR. (c) A 423 bp region of the tha1806 gene was amplified by using Takara LA Taq in the presence of 0 to $0.4 \mathrm{mM}$ ATP. Lanes 1-4,5-8, and 9-12 are the results of the reaction without ttMutS or ttRecA, with $0.8 \mu \mathrm{M}$ ttMutS, and with $0.4 \mu \mathrm{M}$ ttRecA, respectively. The amounts of the amplified fragments were quantified by using the ImageJ software [9] and are shown as bar graphs in the lower panels, where gray and blue indicate nonspecific and desired amplifications, respectively. (d) A $423 \mathrm{bp}$ region of the tha1806 gene was amplified by using Takara LA Taq in the presence of $0.9,2.7,8.0$, or $24 \mathrm{ng} / \mathrm{mL}$ template DNA (T. thermophilus HB8 genomic DNA). The relative amounts of the amplified fragments are shown. Gray and blue bars indicate nonspecific and desired amplifications, respectively.

be amplified. If their error-suppressing effects show different specificities for the target sequences, simultaneous use would cover a wider range of sequences than the single use of ttRecA or ttMutS would. As shown in Figure 2(c), nonspecific amplification upon the amplification of a 466-bp region of the tha1300 gene was effectively suppressed by ttRecA but not by ttMutS. In contrast, ttMutS showed a strong errorsuppressing effect on the amplification of a 1,278-bp region of the ttha1548 gene, whereas ttRecA did not (Figure 2(d)). These results clearly indicate that ttRecA and ttMutS have distinct specificities against the target sequences. This is expected because ttRecA and ttMutS suppress nonspecific amplification in different ways.

\section{Conclusions}

In this study, we clarified that ttMutS and ttRecA do not preclude each other and there are some sequences for which only one of the 2 proteins efficiently suppresses nonspecific amplification. Our results in this study suggest that simultaneous use of ttMutS and ttRecA is better than the use of either ttMutS or ttRecA alone. 

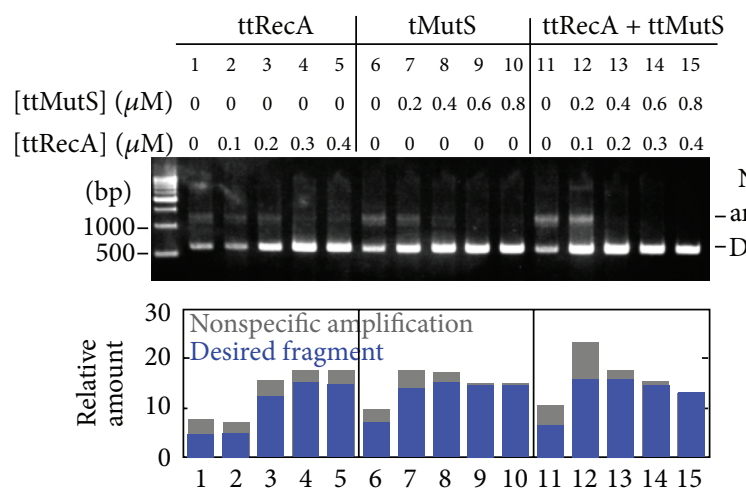

(a)
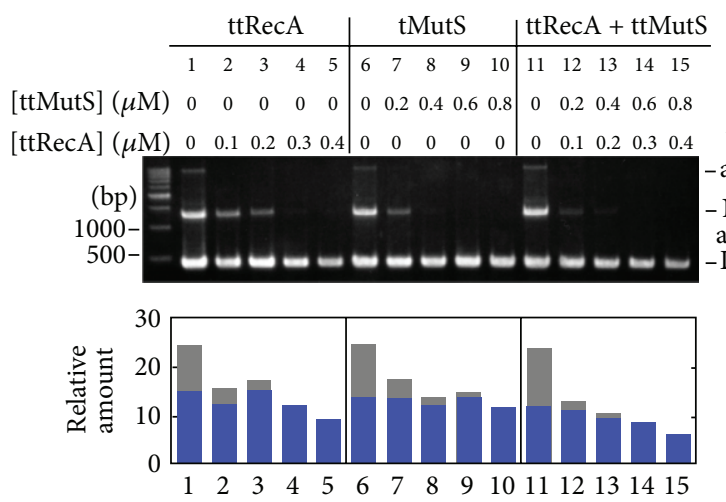

(b)

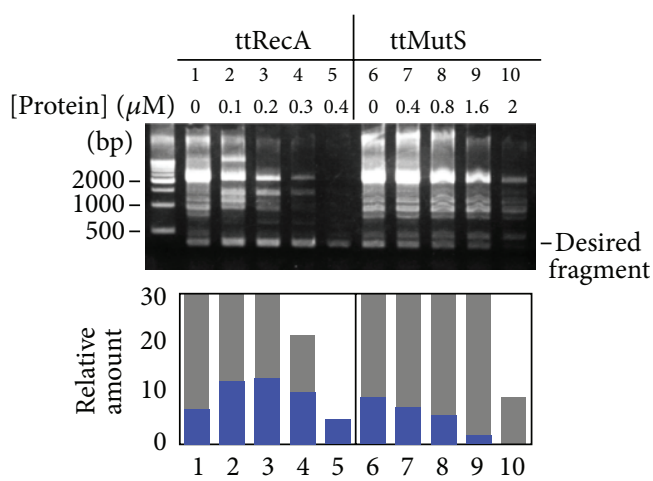

(c)
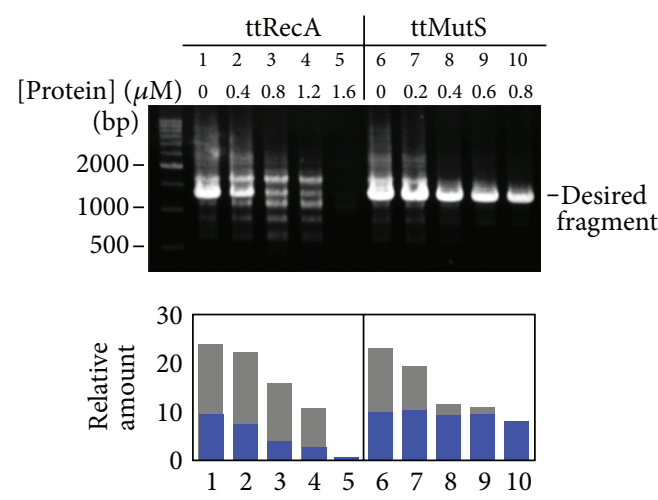

(d)

Figure 2: Various target sequences were amplified in the presence of ttRecA and ttMutS. (a) A 611 bp region of the ttha0122 gene was amplified from T. thermophilus HB8 genomic DNA template in the presence of ttRecA and ttMutS. The amounts of amplified fragments were quantified and are shown as bar graphs in the lower panel. Gray and blue bars indicate nonspecific and desired amplifications, respectively. (b) A 423 bp region of the tha1806 gene was amplified from T. thermophilus HB8 genomic DNA template in the presence of ttRecA and ttMutS. (c) A $466 \mathrm{bp}$ region of the tha1300 gene was amplified from T. thermophilus HB8 genomic DNA in the presence of ttRecA or ttMutS. Note that relatively high concentrations of ttMutS were used here. (d) A 1,278 bp region of the tha1548 gene was amplified in the presence of ttRecA or $\mathrm{ttMutS}$. Note that relatively high concentrations of ttRecA were used here.

\section{Conflict of Interests}

The publication of this paper may affect the value of the patent for the methodology described in our previously published paper [6].

\section{Acknowledgments}

The authors express great thanks to Dr. Yoshitaka Bessho for his valuable discussions, to Dr. Ryoji Masui (Osaka University) and Tomofumi Sakai (Osaka University) for overexpression and purification of ttRecA, and to Kayoko Matsumoto (RIKEN SPring-8 Center) for her excellent help in overexpression of ttMutS. This work was supported by Management Expenses Grants from the government to RIKEN.

\section{References}

[1] K. B. Mullis and F. A. Faloona, "Specific synthesis of DNA in vitro via a polymerase-catalyzed chain reaction," Methods in Enzymology, vol. 155, pp. 335-350, 1987.
[2] R. K. Saiki, S. Scharf, F. Faloona et al., "Enzymatic amplification of $\beta$-globin genomic sequences and restriction site analysis for diagnosis of sickle cell anemia," Science, vol. 230, no. 4732, pp. 1350-1354, 1985.

[3] Y. Mitani, A. Lezhava, Y. Kawai et al., "Rapid SNP diagnostics using asymmetric isothermal amplification and a new mismatch-suppression technology," Nature Methods, vol. 4, no. 3, pp. 257-262, 2007.

[4] K. Fukui, Y. Bessho, A. Shimada, S. Yokoyama, and S. Kuramitsu, "Thermostable mismatch-recognizing protein MutS suppresses nonspecific amplification during polymerase chain reaction (PCR)," International Journal of Molecular Sciences, vol. 14, pp. 6436-6453, 2013.

[5] R. R. Lyer, A. Pluciennik, V. Burdett, and P. L. Modrich, "DNA mismatch repair: functions and mechanisms," Chemical Reviews, vol. 106, no. 2, pp. 302-323, 2006.

[6] K. Fukui, "DNA mismatch repair in eukaryotes and bacteria," Journal of Nucleic Acids, vol. 2010, Article ID 260512, 16 pages, 2010.

[7] Y. Shigemori, T. Mikawa, T. Shibata, and M. Oishi, "Multiplex PCR: use of heat-stable Thermus thermophilus RecA protein to minimize non-specific PCR products," Nucleic Acids Research, vol. 33, no. 14, article e126, 2005. 
[8] S. C. Kowalczykowski, D. A. Dixon, A. K. Eggleston, S. D. Lauder, and W. M. Rehrauer, "Biochemistry of homologous recombination in Escherichia coli," Microbiological Reviews, vol. 58, no. 3, pp. 401-465, 1994.

[9] C. A. Schneider, W. S. Rasband, K. W. Eliceiri et al., "NIH image to imageJ: 25 years of image analysis," Nature Methods, vol. 9, no. 7, pp. 671-675, 2012.

[10] S. Gradia, D. Subramanian, T. Wilson et al., "hMSH2-hMSH6 forms a hydrolysis-independent sliding clamp on mismatched DNA," Molecular Cell, vol. 3, no. 2, pp. 255-261, 1999.

[11] T. Horii, T. Ogawa, and H. Ogawa, "Organization of the recA gene of Escherichia coli," Proceedings of the National Academy of Sciences of the United States of America, vol. 77, no. 1, pp. 313-317, 1980.

[12] R. Masui, T. Mikawa, and S. Kuramitsu, "Local folding of the N-terminal domain of Escherichia coli recA controls proteinprotein interaction," Journal of Biological Chemistry, vol. 272, no. 44, pp. 27707-27715, 1997.

[13] T. Shibata, T. Nishinaka, T. Mikawa et al., "Homologous genetic recombination as an intrinsic dynamic property of a DNA structure induced by recA/rad51-family proteins: a possible advantage of DNA over RNA as genomic material," Proceedings of the National Academy of Sciences of the United States of America, vol. 98, no. 15, pp. 8425-8432, 2001.

[14] G. Obmolova, C. Ban, P. Hsieh, and W. Yang, "Crystal structures of mismatch repair protein MutS and its complex with a substrate DNA," Nature, vol. 407, no. 6805, pp. 703-710, 2000.

[15] M. H. Lamers, A. Perrakis, J. H. Enzlin, H. H. Winterwerp, N. de Wind, and T. K. Sixma, "The crystal structure of DNA mismatch repair protein MutS binding to a G.T mismatch," Nature, vol. 407, no. 6805, pp. 711-717, 2000.

[16] S. Acharya, P. L. Foster, P. Brooks, and R. Fishel, "The coordinated functions of the E. coli MutS and MutL proteins in mismatch repair," Molecular Cell, vol. 12, no. 1, pp. 233-246, 2003. 

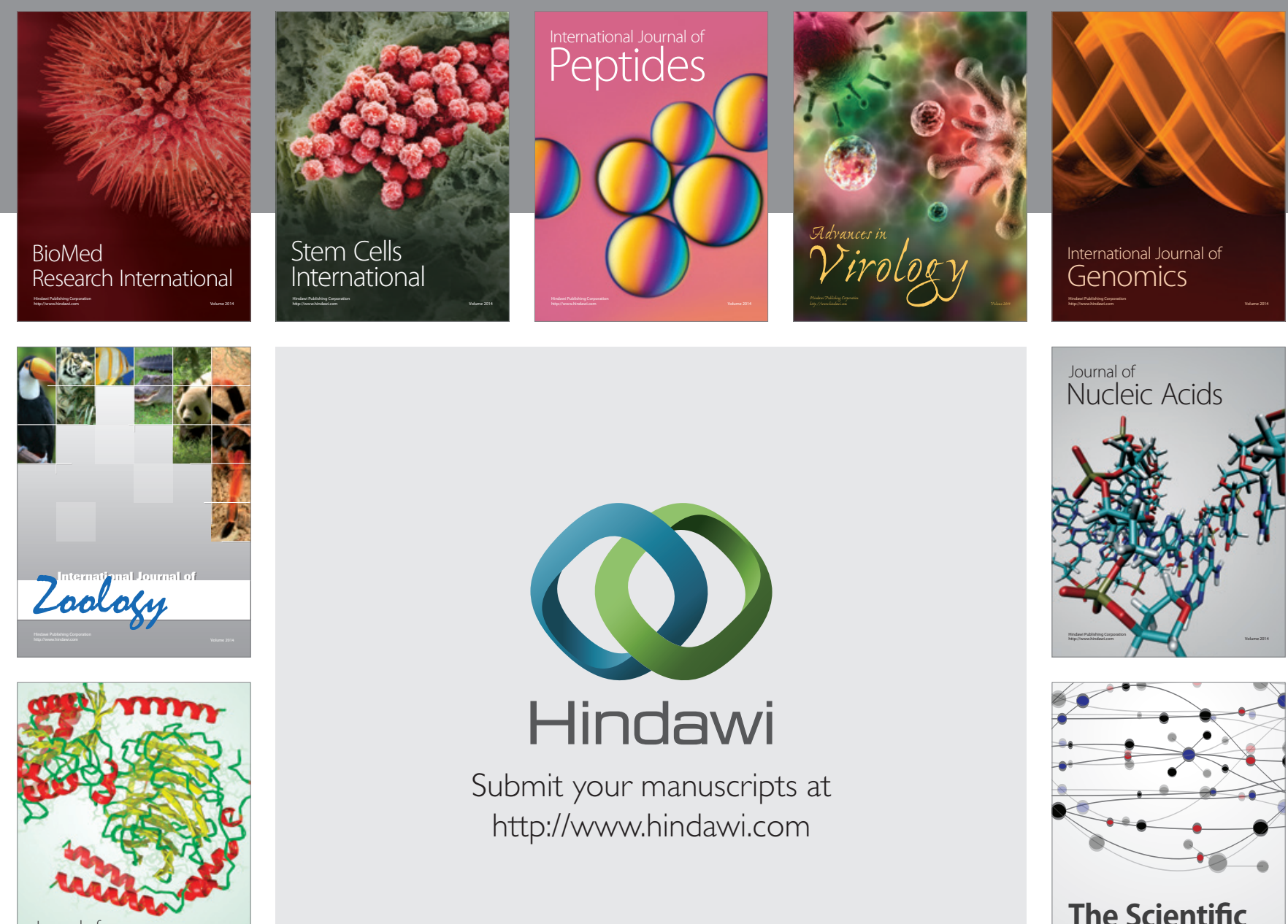

Submit your manuscripts at

http://www.hindawi.com

Journal of
Signal Transduction
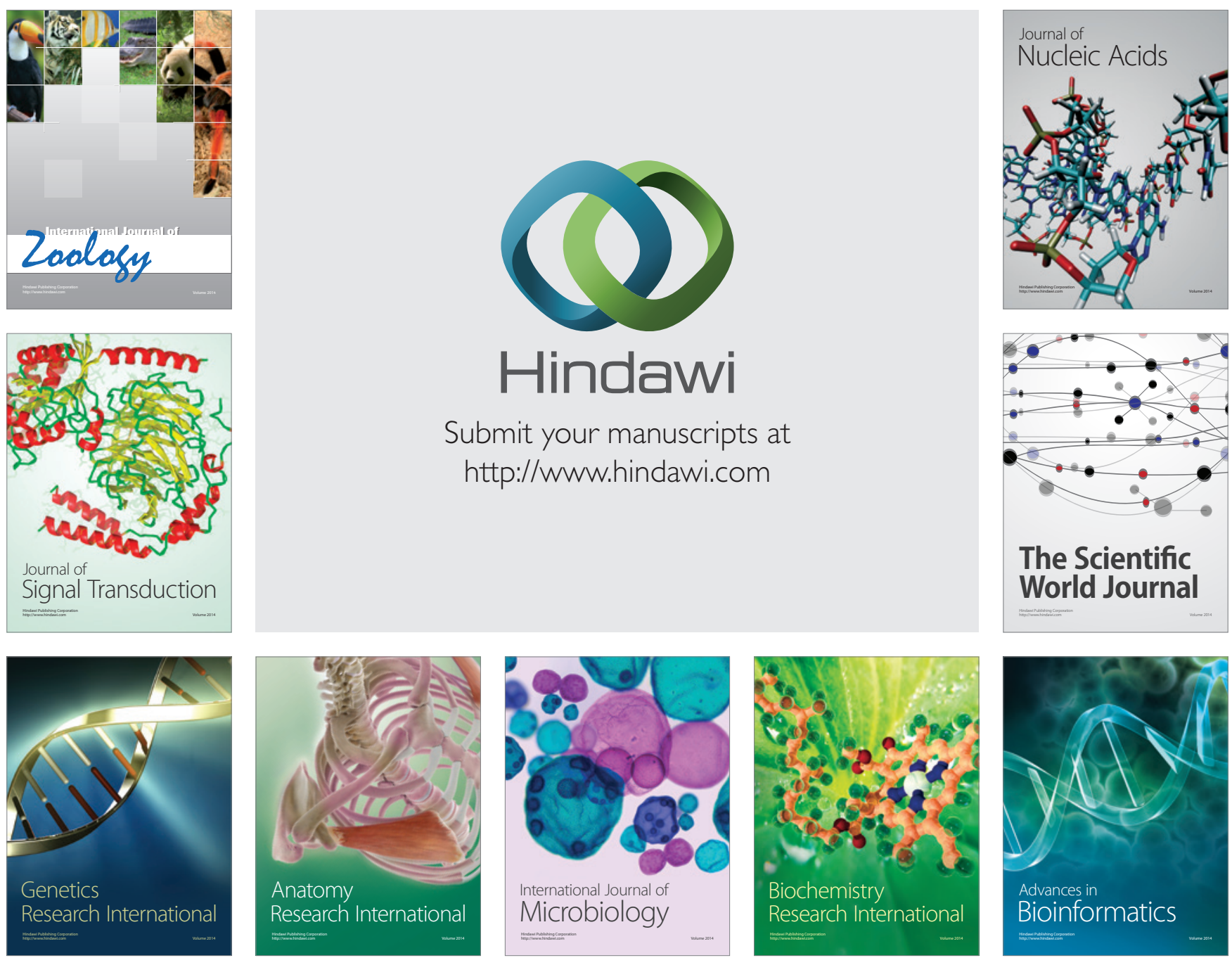

The Scientific World Journal
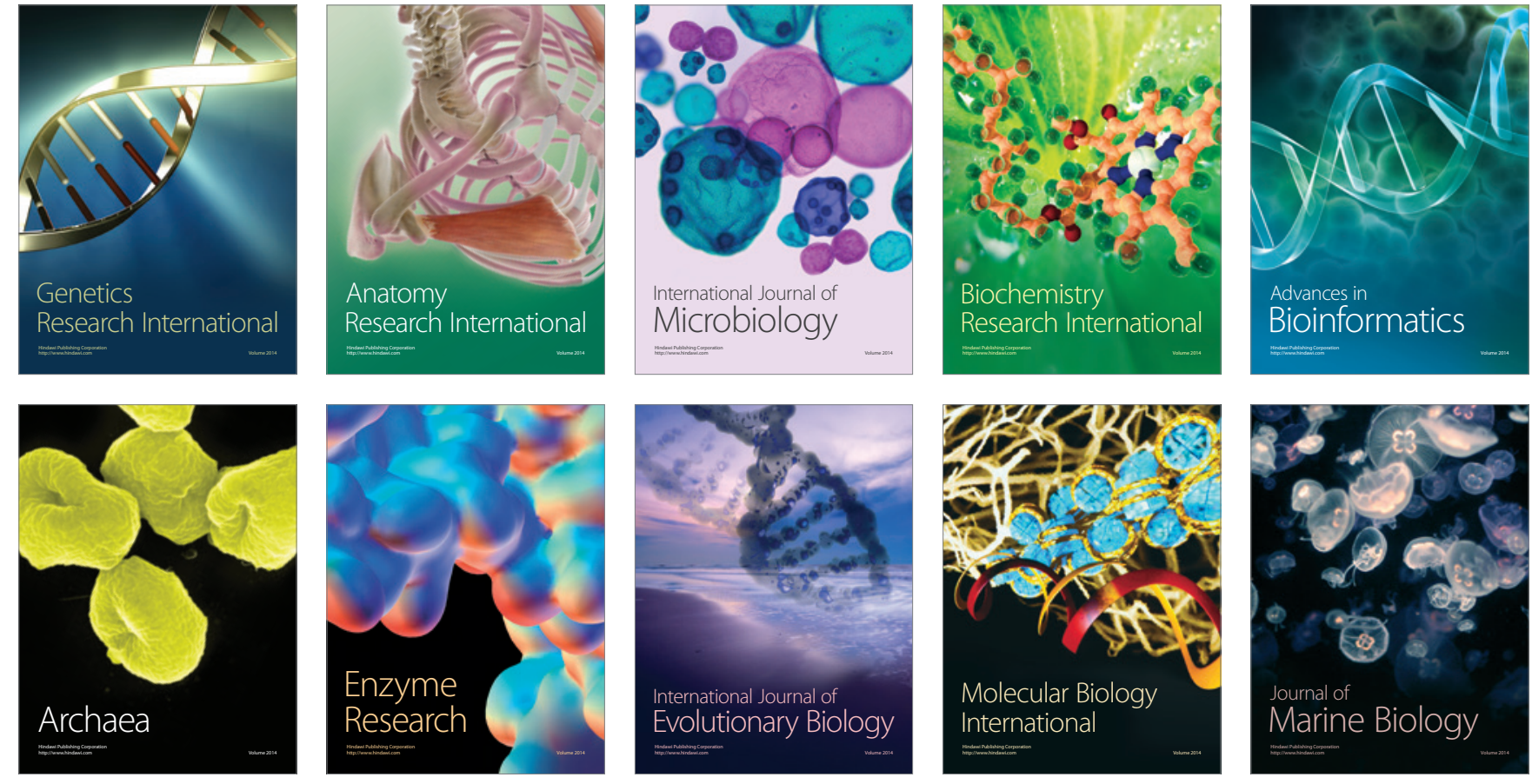